\title{
Collision Energy Evolution of Elliptic and Triangular Flow in a Hybrid Model
}

\author{
J. Auvinen* \\ Frankfurt Institute for Advanced Studies (FIAS) \\ Ruth-Moufang-Strasse 1 \\ D-60438 Frankfurt am Main, Germany \\ E-mail: auvinendfias.uni-frankfurt.de \\ H. Petersen \\ Frankfurt Institute for Advanced Studies (FIAS) \\ Ruth-Moufang-Strasse 1 \\ D-60438 Frankfurt am Main, Germany \\ E-mail: petersen@fias.uni-frankfurt.de
}

\begin{abstract}
While the existence of a strongly interacting state of matter, known as "quark-gluon plasma" (QGP), has been established in heavy ion collision experiments in the past decade, the task remains to map out the transition from the hadronic matter to the QGP. This is done by measuring the dependence of key observables (such as particle suppression and elliptic flow) on the collision energy of the heavy ions. This procedure, known as "beam energy scan", has been most recently performed at the Relativistic Heavy Ion Collider (RHIC).

Utilizing a Boltzmann+hydrodynamics hybrid model, we study the collision energy dependence of initial state eccentricities and the final state elliptic and triangular flow. This approach is well suited to investigate the relative importance of hydrodynamics and hadron transport at different collision energies.
\end{abstract}

CPOD 2013 - 8th International Workshop on Critical Point and Onset of Deconfinement March 11-15

Napa, $C A$

\footnotetext{
* Speaker.
} 


\section{Introduction}

The RHIC beam energy scan program was launched in 2010 to study the features of the QCD phase diagram. In particular, the goal is to search for signs of the possible 1st-order phase transition between the confined and deconfined matter, and locate the critical point marking the boundary of cross-over and 1st-order phase transition in the plane of baryochemical potential $\mu_{B}$ and temperature $T$ [1], predicted by lattice calculations [2].

Elliptic flow is one of the key observables that supports the finding of a strongly coupled quarkgluon plasma at the highest energies of RHIC and the Large Hadron Collider (LHC). Therefore, one would naively expect the elliptic flow to decrease at lower beam energies where the hydrodynamic phase gets shortened or the QGP is not created at all. It has been found, however, that the inclusive charged hadron elliptic flow $v_{2}$ demonstrates very little dependence on the collision energy between $\sqrt{s_{N N}}=7.7-39 \mathrm{GeV}[3]$.

The beam energy dependence of the collective flow has been recently studied with several different models [4]. One possible method for investigating the importance of the hydrodynamical evolution for the flow production is the hybrid approach, where a transport model (a microscopic description of the system) is utilized for the non-equilibrium phases at the beginning and the end of a heavy-ion collision event, and a (macroscopic) hydrodynamical description is used to model the hot and dense stage and the phase transition between the QGP and hadronic matter.

As such a hybrid model should be able to naturally produce the transition from the high-energy heavy ion collisions, with negligible net-baryon density and a large hydrodynamically evolving medium, to smaller energies with finite net-baryon density and lower temperatures, where no such medium is formed, this framework seems optimal for studying the beam energy dependence of the elliptic and triangular flow.

\section{Hybrid model}

This study was performed using a transport + hydrodynamics hybrid model described in [5]. In this approach, the initial state is produced by the Ultrarelativistic Quantum Molecular Dynamics (UrQMD) string / hadronic cascade [6]. The hydrodynamical evolution starts, when the two colliding nuclei have passed through each other: $t_{\text {start }}=\max \left\{\frac{2 R}{\sqrt{\gamma_{C M}^{2}-1}}, 0.5 \mathrm{fm}\right\}$, where $R$ represents the nuclear radius and $\gamma_{C M}=\frac{1}{\sqrt{1-v_{C M}^{2}}}$ is the Lorentz factor. The minimum time of $0.5 \mathrm{fm}$ is chosen based on the hybrid model results at the collision energy $\sqrt{s_{N N}}=200 \mathrm{GeV}$ [7]. At this time, the energy-, momentum- and baryon number densities of the particles, represented by 3D Gaussian distributions that are Lorentz-contracted in the beam direction, are mapped onto the hydro grid. The width parameter of these Gaussians is $\sigma=1.0 \mathrm{fm}$, to preserve the event-by-event initial state fluctuations. Spectators do not participate on the hydrodynamical evolution, but are propagated separately in the cascade.

The model utilizes (3+1)-D ideal hydrodynamics, solving the evolution equations using the SHASTA algorithm [8]. The equation of state is based on a chiral model, coupled to Polyakov loop to include the deconfinement phase transition $[9,10]$, which qualitatively agrees with the lattice QCD data at $\mu_{B}=0$ and is also applicable at finite baryon densities. After the last step of the hydrodynamical evolution, the active EoS is changed from the deconfinement EoS to the hadron 
gas EoS, to ensure that the active degrees of freedom on both sides of the transition hypersurface are exactly equivalent [9].

The transition from hydro to transport ("particlization") is done when the energy density $\varepsilon$ is smaller than the critical value $2 \varepsilon_{0}$, where $\varepsilon_{0}=146 \mathrm{MeV} / \mathrm{fm}^{3}$ represents the nuclear ground state energy density. This corresponds roughly to a switching temperature $T \approx 154 \mathrm{MeV}$ at $\sqrt{s_{N N}}=200$ $\mathrm{GeV} \mathrm{Au+Au}$ collisions [11]. The switching criterion with respect to the energy density is kept constant over all beam energies in this study, but naturally corresponds to different combinations of temperature and baryochemical potential at different values of $\sqrt{s_{N N}}$.

From the iso-energy density hypersurface, constructed using the Cornelius algorithm [11], particle distributions are generated according to the Cooper-Frye formula. After the particlization, rescatterings and final decays are computed in the UrQMD. The end result is a distribution of particles which can be directly compared against the experimental data.

This hybrid approach has the advantage of dynamically changing the importance of the nonequilibrium transport and the hydrodynamic part of the evolution and involves a proper equation of state that is applicable at high net baryon densities. The high viscosity during the hadron gas evolution is taken into account, while the small viscosity during the hydrodynamic evolution has been neglected for simplicity to demonstrate qualitative behavior.

\section{Results}

\subsection{Particle spectra}

Before going into more detailed observables such as the elliptic flow, we check how well the hybrid model reproduces the more general features of the system, such as the particle spectra. The evolution of $m_{T}$ spectra at midrapidity $|y|<0.5$ for $\pi^{-}, K^{+}$and $K^{-}$as a function of beam energy in $\mathrm{Pb}+\mathrm{Pb}$-collisions is illustrated in Figure 1. For the beam energy $E_{\text {lab }}=40 \mathrm{AGeV}$, corresponding to the collision energy $\sqrt{s_{N N}} \approx 9 \mathrm{GeV}$, there is a good agreement with the NA49 data [12]. However, at the higher energies it becomes clear that the pion slope is a little too flat and there is an excess of kaons produced. This necessitates revisiting the model parameters, chiefly the value of particlization energy density, in the future studies. Nevertheless, for the purpose of the current investigation the agreement with the experimental data is sufficient.

\subsection{Elliptic flow}

Our primary interest here is to see, if the insensitivity of the elliptic flow $v_{2}$ on the collision energy can be understood within the hybrid approach. In this study $v_{2}$ is computed from the particle momentum distributions using the event plane method [13]. This and the new implementation of the Cooper-Frye hypersurface finder and particlization are the main differences in this calculation compared to previous studies of elliptic flow in the same hybrid approach [14].

Figure 2 shows the produced $p_{T}$-integrated elliptic flow $v_{2}$ in $\mathrm{Au}+\mathrm{Au}$-collisions, compared with the STAR data for three centrality classes: $(0-5) \%,(20-30) \%$ and (30-40)\%. In the model these are respectively represented by the impact parameter intervals $b=0-3.4 \mathrm{fm}, b=6.7-8.2$ $\mathrm{fm}$ and $b=8.2-9.4 \mathrm{fm}$, based on the optical Glauber model estimates [15].

The agreement with the experimental data in the most central collisions is good above $\sqrt{s_{N N}}=$ $11.5 \mathrm{GeV}$; at the lowest energies the model appears to produce more flow than is observed in the 
a) $E_{\text {lab }}=40 \mathrm{~A} \mathrm{GeV} m_{\mathrm{T}}$ distribution at $|\mathrm{y}|<0.5$

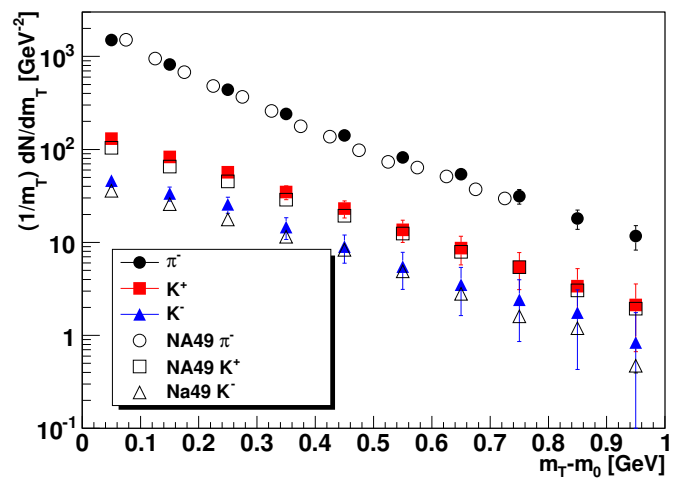

b) $E_{\text {lab }}=158 \mathrm{~A} \mathrm{GeV} \mathrm{m}_{\mathrm{T}}$ distribution at $|\mathrm{y}|<0.5$

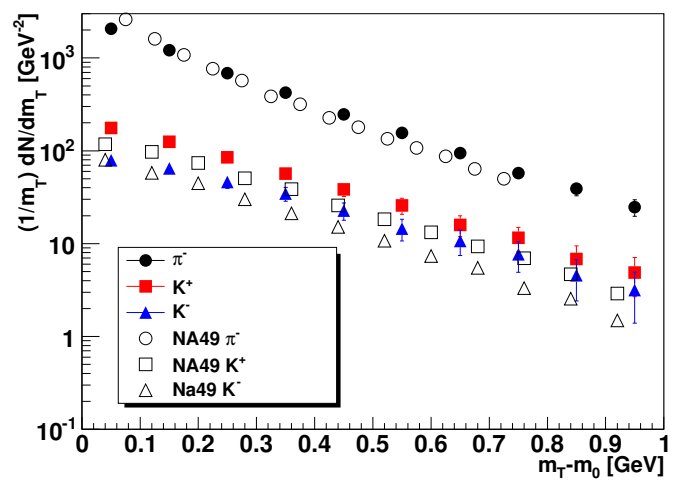

Figure 1: Transverse mass spectra at midrapidity $|y|<0.5$ for $\pi^{-}, K^{+}$and $K^{-}$in $\mathrm{Pb}+\mathrm{Pb}$-collisions, compared to the NA49 data [12] at beam energy a) $E_{\text {lab }}=40 \mathrm{AGeV}$, b) $E_{\text {lab }}=158 \mathrm{AGeV}$.

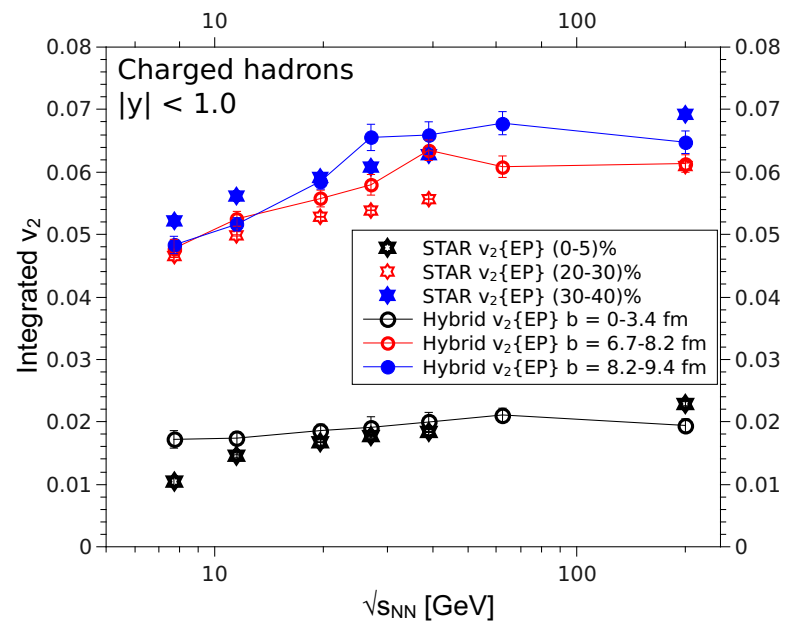

Figure 2: Integrated elliptic flow $v_{2}$ at midrapidity $|y|<1.0$ in $\mathrm{Au}+\mathrm{Au}$-collisions, for collision energies $\sqrt{s_{N N}}=7.7-200 \mathrm{GeV}$ and three different impact parameter ranges, compared with the STAR data [3, 16].

experiments. However, in midcentral collisions the hybrid model does reproduce the measured increase of $v_{2}$ with respect to $\sqrt{s_{N N}}$.

We can now investigate in more detail the contribution to $v_{2}$ from different phases of the heavy ion collision event. Figure 3 demonstrates the magnitude of $v_{2}$ before the hydrodynamical evolution, right after particlization and finally after the hadronic rescatterings performed in the UrQMD (the end result). In the most central collisions, where the overall elliptic flow is small compared to mid-central collisions, the effect of the hadronic rescatterings is negligible. In the impact parameter range $b=8.2-9.4 \mathrm{fm}$ the contribution from the hadronic rescatterings is about $10 \%$.

In both centralities, it is observed that at $\sqrt{s_{N N}}=7.7 \mathrm{GeV}$, hydrodynamics contribute very little to the elliptic flow; for the mid-central collisions, $v_{2}$ is in practice completely produced by the transport dynamics. However, already at $\sqrt{s_{N N}}=11.5 \mathrm{GeV}$ the contribution from the hydrodynamic phase is significant. 

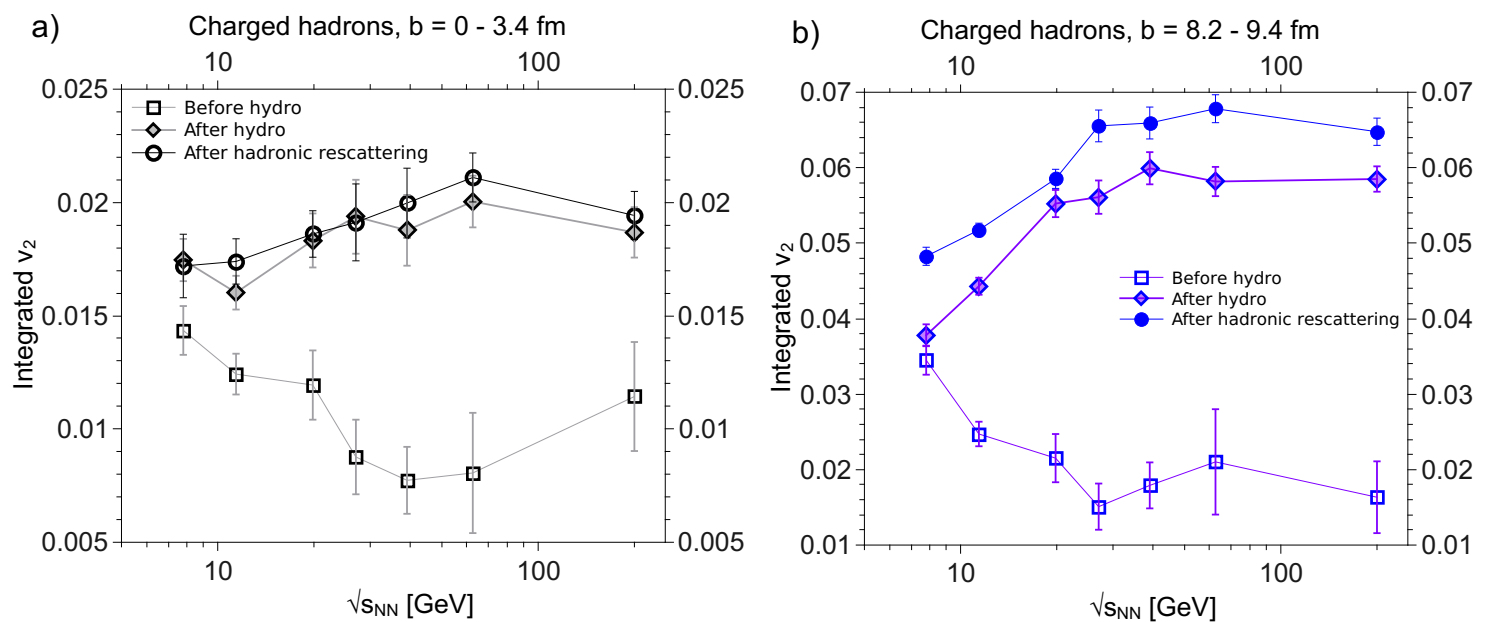

Figure 3: Magnitude of $v_{2}$ at the beginning of hydrodynamical evolution (squares), immediately after particlization (diamonds) and after the full simulation (circles, the same as in Fig. 2) at a) central collisions and b) midcentral collisions.

As seen in Figure 4, $v_{2}\left(p_{T}\right)$ produced by the hybrid model systematically overshoots the data at all collision energies. This suggests the need for either adding viscous effects or stopping the hydrodynamical evolution earlier at a higher energy density. The dependence on the collision energy is non-existent, which is in accord with the STAR data, and is in this framework understood as the non-equilibrium hadron dynamics compensating for the shortened hydrodynamical evolution at lower $\sqrt{s_{N N}}$.

\subsection{Triangular flow}

The triangular flow $v_{3}$ originates solely from the event-by-event variations in the initial configuration of the colliding nucleons, and is thus a good observable for measuring the system sensitivity to the initial state fluctuations. In the most central collisions, integrated $v_{3}$ increases from 0.005 to above 0.01 with increasing collision energy (see Fig. 5a), whereas in midcentrality there is a rapid rise from $\approx 0$ at $\sqrt{s_{N N}}<10 \mathrm{GeV}$ to the value of $\approx 0.015-0.02$ for $\sqrt{s_{N N}} \geq 19.6 \mathrm{GeV}$. This behavior is reflected also on $v_{3}\left(p_{T}\right)$ in Fig. $5 \mathrm{~b}$. The energy dependence of $v_{3}$ is very similar to what was seen for the hydrodynamically produced $v_{2}$ in Figure $3 \mathrm{~b}$, suggesting that in this case the transport part of the model is unable to compensate for the diminished hydro phase.

The magnitude of triangular flow at $\sqrt{s_{N N}}=200 \mathrm{GeV}$ is close to the measured value for both centralities [17]. However, the decrease to zero at low energies is not supported by the preliminary STAR data, where very little $\sqrt{s_{N N}}$-dependence is seen below $30 \mathrm{GeV}$ and the rise begins only at later energies [18].

\subsection{Effect of initial geometry}

Figure 6a illustrates the collision energy and centrality dependencies of the average initial state spatial eccentricity $\left\langle\varepsilon_{2}\right\rangle$ and triangularity $\left\langle\varepsilon_{3}\right\rangle$. The eccentricity and triangularity in an event are defined as in [19] and calculated at the beginning of hydrodynamical evolution $t_{\text {start }}$. 
a) $\quad{ }_{\mathrm{S}_{\mathrm{NN}}}=7.7 \mathrm{GeV}, \mathrm{b}=6.7-8.2 \mathrm{fm}$

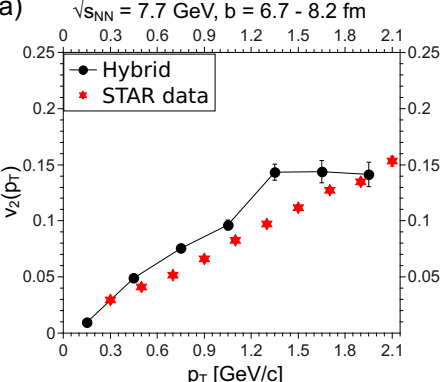

d) $\quad V_{\mathrm{S}_{\mathrm{NN}}}=27 \mathrm{GeV}, \mathrm{b}=6.7-8.2 \mathrm{fm}$

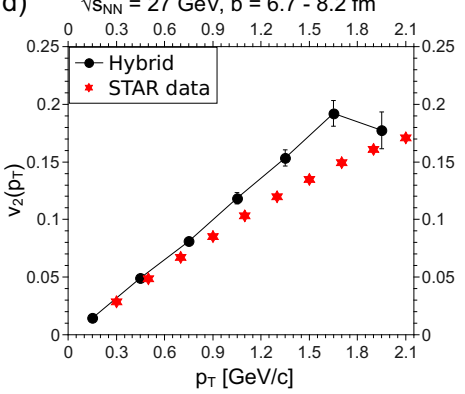

b) $\quad \sqrt{\mathrm{S}_{\mathrm{NN}}}=11.5 \mathrm{GeV}, \mathrm{b}=6.7-8.2 \mathrm{fm}$

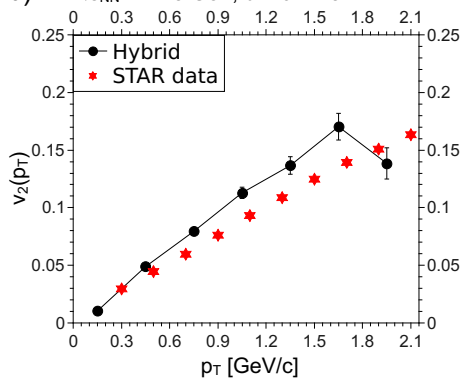

e) $\quad V_{\mathrm{S}_{\mathrm{NN}}}=39 \mathrm{GeV}, \mathrm{b}=6.7-8.2 \mathrm{fm}$

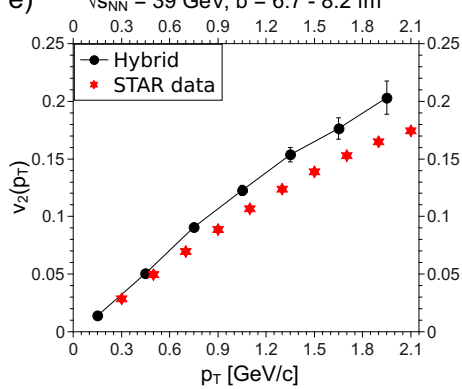

c) $\quad{ }_{\mathrm{S}_{\mathrm{NN}}}=19.6 \mathrm{GeV}, \mathrm{b}=6.7-8.2 \mathrm{fm}$

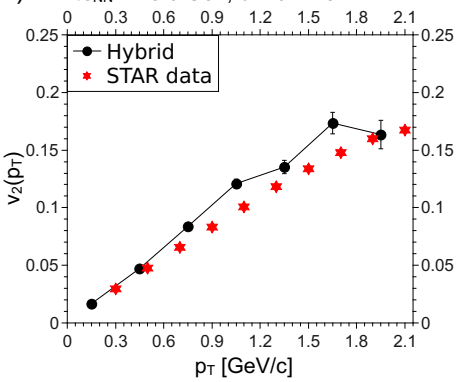

f) $\quad{ }_{\mathrm{S}_{\mathrm{NN}}}=7.7-39 \mathrm{GeV}, \mathrm{b}=6.7-8.2 \mathrm{fm}$

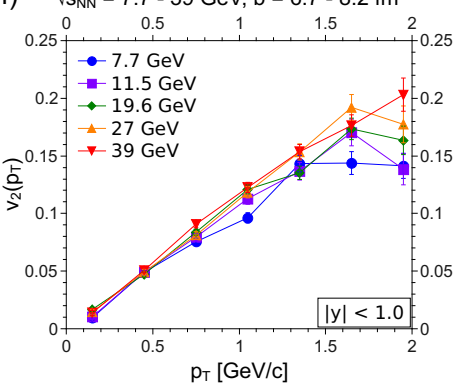

Figure 4: (a-e) Differential $v_{2}$ at midrapidity $|y|<1.0$ for collision energies $\sqrt{s_{N N}}=7.7-39 \mathrm{GeV}$ in impact parameter range $b=6.7-8.2 \mathrm{fm}$, compared with the STAR data in (20-30)\% centrality [3]. Panel (f): compilation of hybrid model $v_{2}\left(p_{T}\right)$ results from panels (a-e).
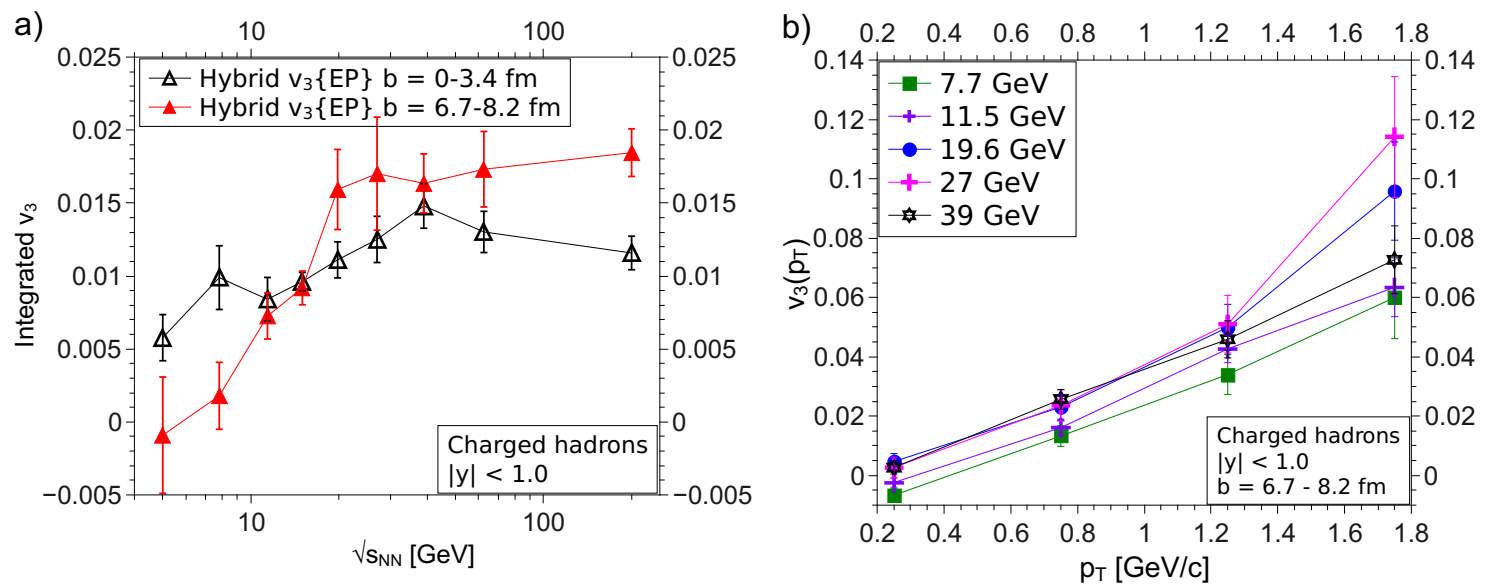

Figure 5: a) Integrated $v_{3}$ at midrapidity $|y|<1.0$ in central collisions $(b=0-3.4 \mathrm{fm}$, open triangles) and midcentral collisions ( $b=6.7-8.2 \mathrm{fm}$, solid triangles). b) $v_{3}\left(p_{T}\right)$ in midcentral collisions.

In the most central collisions, both the average eccentricity and triangularity are similar in magnitude. The situation changes at mid-central collisions, where, due to the collision geometry, $\left\langle\varepsilon_{2}\right\rangle$ is clearly larger than $\left\langle\varepsilon_{3}\right\rangle$. There is only a weak dependence on the collision energy. This is not surprising, as neither the typical binary collision spatial distribution nor the inelastic nucleonnucleon cross section $\sigma_{N N}$ are expected to change significantly within the examined energy range. What does change rapidly at lower collision energies is $t_{\mathrm{star}}$, which drops from $3.22 \mathrm{fm}$ at $\sqrt{s_{N N}}=$ $7.7 \mathrm{GeV}$ to $1.23 \mathrm{fm}$ at $\sqrt{s_{N N}}=19.6 \mathrm{fm}$. This longer transport evolution would thus be the main 

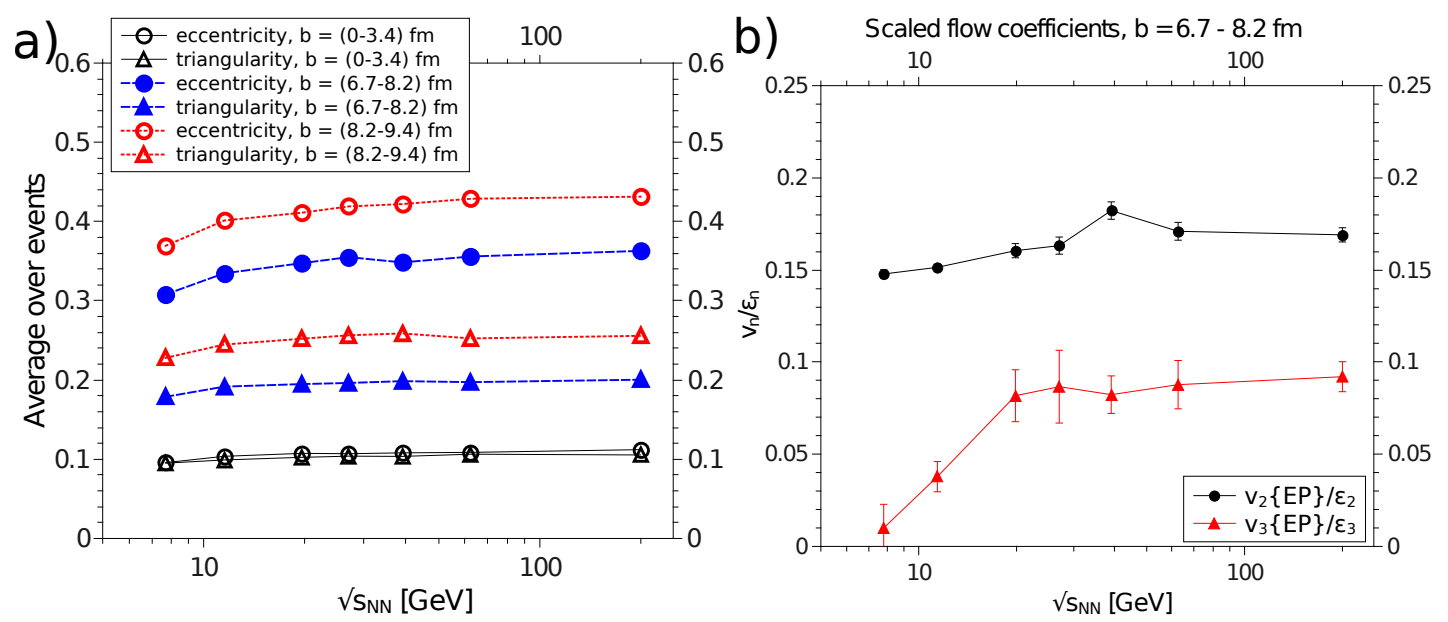

Figure 6: a) Average eccentricity $\left\langle\varepsilon_{2}\right\rangle$ (circles) and triangularity $\left\langle\varepsilon_{3}\right\rangle$ (triangles) as a function of collision energy $\sqrt{s_{N N}}$, for impact parameter ranges $b=0-3.4 \mathrm{fm}$ (solid lines), $6.7-8.2 \mathrm{fm}$ (dashed lines) and $8.2-9.4 \mathrm{fm}$ (dotted lines). b) Scaled flow coefficients $v_{2} /\left\langle\varepsilon_{2}\right\rangle$ and $v_{3} /\left\langle\varepsilon_{3}\right\rangle$ for $b=6.7-8.2 \mathrm{fm}$.

reason for the systematic decrease of $\left\langle\varepsilon_{2}\right\rangle$ and $\left\langle\varepsilon_{3}\right\rangle$ at low energies in Figure 6a.

In order to examine the system response to initial geometry, we scale $v_{2}$ and $v_{3}$ with $\left\langle\varepsilon_{2}\right\rangle$ and $\left\langle\varepsilon_{3}\right\rangle$, respectively. The result for $b=6.7-8.2 \mathrm{fm}$ is shown in Figure $6 \mathrm{~b}$. As the initial geometry displays little change over $\sqrt{s_{N N}}$, the result reflects what we already saw with the unscaled flow coefficients: the relation of the elliptic flow to the initial eccentricity remains almost constant for the whole collision energy range, while the $v_{3}$ response to the triangularity of the initial state reaches a constant value only after $19.6 \mathrm{GeV}$. This confirms that compared to hydrodynamics, the string / hadron transport dynamics are inefficient for transforming the initial state spatial fluctuations into the final state momentum anisotropy.

\section{Summary}

In this study, we have demonstrated that it is possible to reproduce the experimentally observed $v_{2}$ by utilizing a hybrid transport + hydrodynamics approach. In such a framework, it is seen that the hadron / string pre-equilibrium dynamics can compensate for the diminished hydrodynamical evolution for $v_{2}$ production at lower collision energies. For the triangular flow $v_{3}$ this is not true, and the system response to triangularity generated by the initial state fluctuations drops to near zero at the collision energies below $10 \mathrm{GeV}$.

However, while the values for the triangular flow $v_{3}$ at high collision energies quantitatively agree with the experimental results, there is a qualitative disagreement with the preliminary STAR data, which display non-zero $v_{3}$ at lower collision energies. As the transport dynamics have been proven ineffective for $v_{3}$ production in this investigation, this would suggest that the hydrodynamically behaving matter is manifested at the lower collision energies in greater extent than expected.

There are also issues with kaon production and $v_{2}\left(p_{T}\right)$ overestimating the data at higher $p_{T}$, which suggest that a slight re-tuning of the model parameters is required for the optimal agreement with the experimental data. These issues are revisited in the near future. 


\section{Acknowledgements}

The authors acknowledge funding of the Helmholtz Young Investigator Group VH-NG-822. Computational resources have been provided by the Center for Scientific Computing (CSC) at the Goethe-University of Frankfurt.

\section{References}

[1] L. Kumar [STAR Collaboration], Nucl. Phys. A 862-863, 125 (2011).

[2] Z. Fodor and S. D. Katz, JHEP 0404, 050 (2004);

S. Ejiri, Phys. Rev. D 78, 074507 (2008);

R. V. Gavai and S. Gupta, Phys. Rev. D 78, 114503 (2008).

[3] L. Adamczyk et al. [STAR Collaboration], Phys. Rev. C 86, 054908 (2012).

[4] V. P. Konchakovski, E. L. Bratkovskaya, W. Cassing, V. D. Toneev, S. A. Voloshin and V. Voronyuk, Phys. Rev. C 85, 044922 (2012);

C. Shen and U. Heinz, Phys. Rev. C 85, 054902 (2012) [Erratum-ibid. C 86, 049903 (2012)];

D. Solanki, P. Sorensen, S. Basu, R. Raniwala and T. K. Nayak, Phys. Lett. B 720, 352 (2013).

[5] H. Petersen, J. Steinheimer, G. Burau, M. Bleicher and H. Stocker, Phys. Rev. C 78, 044901 (2008).

[6] S. A. Bass, M. Belkacem, M. Bleicher, M. Brandstetter, L. Bravina, C. Ernst, L. Gerland and M. Hofmann et al., Prog. Part. Nucl. Phys. 41, 255 (1998);

M. Bleicher, E. Zabrodin, C. Spieles, S. A. Bass, C. Ernst, S. Soff, L. Bravina and M. Belkacem et al., J. Phys. G 25, 1859 (1999).

[7] H. Petersen, C. Coleman-Smith, S. A. Bass and R. Wolpert, J. Phys. G 38, 045102 (2011).

[8] D. H. Rischke, S. Bernard and J. A. Maruhn, Nucl. Phys. A 595, 346 (1995);

D. H. Rischke, Y. Pursun and J. A. Maruhn, Nucl. Phys. A 595, 383 (1995) [Erratum-ibid. A 596, 717 (1996)].

[9] J. Steinheimer, V. Dexheimer, H. Petersen, M. Bleicher, S. Schramm and H. Stoecker, Phys. Rev. C 81, 044913 (2010).

[10] J. Steinheimer, S. Schramm and H. Stocker, J. Phys. G 38, 035001 (2011).

[11] P. Huovinen and H. Petersen, arXiv:1206.3371 [nucl-th].

[12] S. V. Afanasiev et al. [NA49 Collaboration], Phys. Rev. C 66, 054902 (2002).

[13] A. M. Poskanzer and S. A. Voloshin, Phys. Rev. C 58, 1671 (1998); J. -Y. Ollitrault, nucl-ex/9711003.

[14] H. Petersen and M. Bleicher, Phys. Rev. C 79, 054904 (2009);

H. Petersen and M. Bleicher, Phys. Rev. C 81, 044906 (2010).

[15] K. J. Eskola, K. Kajantie and J. Lindfors, Nucl. Phys. B 323, 37 (1989);

D. Miskowiec, http://web-docs.gsi.de/ misko/overlap/

[16] J. Adams et al. [STAR Collaboration], Phys. Rev. C 72, 014904 (2005).

[17] L. Adamczyk et al. [STAR Collaboration], arXiv:1301.2187 [nucl-ex].

[18] Y. Pandit [STAR Collaboration], talk at Quark Matter 2012.

[19] B. Schenke, P. Tribedy and R. Venugopalan, Phys. Rev. C 86, 034908 (2012). 